\title{
Nonrandom X Chromosome DNA Methylation Patterns in Hemophiliac Females
}

\author{
Perry D. Nisen and Pamela G. Waber
}

Department of Pediatrics, Division of Hematology-Oncology, Schneider Children's Hospital of Long Island Jewish Medical Center, New Hyde Park, New York 11042; and Department of Pediatrics, University of Texas Southwestern Medical Center, Dallas, Texas 75235

\begin{abstract}
Molecular $\mathbf{X}$ chromosome inactivation analysis was used to characterize three females (and their families) with severe hemophilia. First, the maternal and paternal $\mathbf{X}$ chromosomes were distinguished by restriction fragment length polymorphisms (RFLPs). Second, the patterns of methylation of $X$ chromosome genes using methylation-sensitive restriction endonucleases were determined. Of the six $X$ chromosome probes tested, only the phosphoglycerol-kinase (PGK) and hypoxanthine-phosphoribosyl-transferase (HPRT) clones were informative, indicating that other $\mathbf{X}$ chromosome probes are not useful for $X$ inactivation analysis. After digestion with Hpa II or Hha I, the hybridization intensity of the RFLPs of all three mothers and an unaffected sister were diminished by $50 \%$, consistent with random $X$ chromosome inactivation. The methylation patterns of the $X$ chromosomes of the affected females, however, were clearly nonrandom. Depending upon the probe and the patient, HPRT and PGK sequences were either completely methylated or unmethylated. These findings are extremely suggestive that nonrandom $X$ chromosome inactivation (lyonization) is the basis for severe hemophilia in these females.
\end{abstract}

\section{Introduction}

Phenotypic expression of $\mathrm{X}$-linked recessive diseases in females is unusual. In one instance, severe hemophilia $B$ in a girl was due to nonrandom $\mathrm{X}$ chromosome inactivation caused by a deletion in one of the $\mathrm{X}$ chromosomes distal to a normal Factor IX allele (1). As a means of assessing the extent of nonrandom X chromosome inactivation in other cytogenetically normal females with hemophilia, we used the technique of molecular X chromosome inactivation analysis. The technique involves the simultaneous detection of restriction fragment length polymorphisms (RFLPs) ${ }^{1}$ and patterns of methyl-

Address correspondence to Dr. Perry D. Nisen, Department of Pediatrics, Division of Hematology-Oncology, University of Texas Southwestern Medical School, 5323 Harry Hines Blvd., Dallas, TX 752359063.

Received for publication 16 November 1988 and in revised form 19 January 1989.

1. Abbreviations used in this paper: RFLP, restriction fragment length polymorphism.

J. Clin. Invest.

(C) The American Society for Clinical Investigation, Inc.

0021-9738/89/04/1400/04 \$2.00

Volume 83, April 1989, 1400-1403 ation of $\mathrm{X}$ chromosome genes using methylation-sensitive restriction endonucleases. $\mathrm{X}$ chromosome inactivation analysis was initially used to establish the clonal origin of human tumors (2) and has also been used to determine carrier status in X-linked agammaglobulinemia (3), Wiscott-Aldrich syndrome (4), and X-linked severe combined immunodeficiency (5). In this report, molecular X chromosome inactivation analysis was used to characterize three unusual families in which one of the daughters sporadically manifested severe hemophilia.

\section{Methods}

Subjects. Two females (families B and C) were diagnosed with severe hemophilia A and one female (family A) was diagnosed with severe hemophilia B by standard measurement of clotting factor activity and antigen levels. None of the other family members had hemophilia, nor was there evidence that the mothers were carriers of the disease. Blood was obtained from these patients and their family members as part of an approved protocol of the Human Subjects Review Committee of the Long Island Jewish Medical Center (New Hyde Park, NY).

DNA isolation, enzyme digestion, electrophoresis, and hybridization. Blood was collected in EDTA tubes. Chromosomal DNA was isolated from white blood cell nuclei as described previously (1). Restriction endonucleases were obtained from New England Biolabs (Beverly, MA), International Biotechnologies, Inc. (New Haven, CT), and Bethesda Research Laboratories (Gaithersburg, MD) and were used according to the conditions specified by the supplier. Agarose gel electrophoresis, Southern transfer to Nytran membranes (Schleicher \& Schuell, Keene, NH), radiolabeling of molecular probes by random priming, and hybridizations were performed as described previously (1).

Molecular probes. FIX (8.13) contains two fragments of human Factor IX genomic sequences: a 2.5-kb fragment containing the d exon of the Factor IX gene and additional adjacent intron sequences (clone 8), and a triple insert of an $0.46-\mathrm{kb}$ fragment that contains most of the $b$ exon, all of the $c$ exon, and contiguous intron sequences (clone 13) (6). FVIII (p114.12) is a genomic clone containing exons 17 and 18 and contiguous introns of the human Factor VIII gene (p114.12) (7). St14 (8) and Dx 13 (9) are random X chromosome DNA probes that are linked to Factor VIII. PGK (pSPT 19.1) is an 0.8-kb fragment of the phosphoglycerol-kinase gene containing the PGK-1 promoter (10). HPRT (pPB 1.7) is a 1.7-kb fragment from the first intron of the human hypoxanthine-phosphoribosyl-transferase gene (11).

Densitometry. The intensity of hybridization was quantitated by densitometric tracing of duplicate autoradiograms using a quantimet densitometer (Cambridge Instruments, Inc., Ossining, NY) (12).

\section{Results}

Methylation patterns of PGK and HPRT have been extensively used for $\mathrm{X}$ chromosome inactivation analysis. In the process of characterizing our patients by $\mathrm{X}$ chromosome inac- 


\begin{tabular}{|c|c|c|c|c|c|c|c|c|c|c|c|}
\hline Probe & Enzyme & $O$ & $\begin{array}{r}A \\
-\square\end{array}$ & & $O L$ & $\begin{array}{r}\text { B } \\
\square\end{array}$ & & $\mathrm{O}$ & $-\square$ & 0 & 0 \\
\hline FIX (8.13) & Taq I & $1.3 / 1.3$ & 1.8 & $1.3 / 1.8$ & $1.8 / 1.8$ & 1.8 & $1.8 / 1.8$ & $1.8 / 1.8$ & 1.8 & $1.8 / 1.8$ & $1.8 / 1.8$ \\
\hline & Xmn I & $6.5,5 / 6.5,5$ & 11.5 & $6.5,5 / 11.5$ & $11.5 / 11.5$ & 11.5 & $11.5 / 11.5$ & $11.5 / 11.5$ & 11.5 & $11.5 / 11.5$ & $11.5 / 11.5$ \\
\hline FVIII exon 17 & Bcl I & $0.86 / 1.17$ & 1.17 & $0.86 / \mathrm{del}$ & $0.86 / 1.17$ & 1.17 & $0.86 / 1.17$ & $0.86 / 1.17$ & 1.17 & $0.86 / 1.17$ & $0.86 / 1.17$ \\
\hline St 14* & Taq I & $1 / 2$ & 3 & $1 / \mathrm{del}$ & $1 / 3$ & 2 & $1 / 2$ & $1 / 1$ & 2 & $1 / 2$ & $1 / 2$ \\
\hline Dx13 & Bgl II & ND & ND & ND & $3.0 / 6.5$ & 6.5 & $3.0 / 6.5$ & $6.5 / 6.5$ & 3.0 & $3.0 / 6.5$ & $3.0 / 6.5$ \\
\hline PGK & Bst XI, Pst I & $0.9 / 1.05$ & 1.05 & $0.9 / 1.05$ & $0.9 / 1.05$ & 1.05 & $0.9 / 1.05$ & $0.9 / 1.05$ & 1.05 & $0.9 / 1.05$ & 1.05/1.05 \\
\hline HPRT & Bam HI & $12 / 25$ & 25 & $12 / 25$ & $25 / 25$ & 12 & $25 / 12$ & $25 / 25$ & 12 & $25 / 12$ & $25 / 12$ \\
\hline & Bam HI, Pvu II & $12 / 18$ & 18 & $12 / 18$ & $18 / 18$ & 12 & $18 / 12$ & $18 / 18$ & 12 & $25 / 18$ & $25 / 18$ \\
\hline Karyotype & & $46, X X$ & $46, X Y$ & $\begin{array}{l}46, X X \\
\operatorname{del}(X) \\
\text { (q27) }\end{array}$ & ND & ND & $46, X X$ & ND & ND & $46, X X$ & ND \\
\hline
\end{tabular}

The numbers refer to the kilobase size of the RFLPs which were determined by hybridization of the indicated DNA probes to Southern blots of chromosomal DNAs digested with the restriction endonucleases indicated. Alleles are separated by the symbol /. Pedigrees of the three families are designated A, B, and C. Open circle, unaffected female; open square, unaffected male; closed circle, affected female (family A is hemophilia B and families B and C are hemophilia A); ND, not determined. The karyotype, when known, is listed. * For St 14, complex patterns involving multiple bands are obtained, designated by the numbers indicated for each allele. Boldface numbers indicate informative RFLPs.

tivation analysis, we sought to identify other $\mathrm{X}$ chromosome probes that could be similarly used for this type of study.

The first step was to identify RFLPs to distinguish the maternal and paternal $\mathrm{X}$ chromosomes. The results are summarized in Table I. Two females with severe hemophilia A (families B and C) had heterozygous RFLPs with three different Factor VIII probes, PGK, and HPRT. The previously described female with hemophilia B (family A) (and a paternal del Xq27 $\rightarrow$ ter) (1) was heterozygous for Factor IX, HPRT, and PGK.

The second step was to determine the patterns of methylation for the $\mathrm{X}$ chromosomes by differential sensitivity to digestion with the methylation-sensitive restriction endonucleases Hpa II or Hha I. Only the PGK (Fig. 1) and HPRT (Fig. 2) probes were informative. RFLPs of the other X chromosome DNA probes listed in Table I either completely disappeared or persisted after digestion with Hha I or Hpa II (not shown). The signal intensity of the RFLP bands of the mothers and an unaffected sister were diminished by $50 \%$, consistent with random X chromosome inactivation. The RFLP patterns of the affected females, however, were different: the female with hemophilia B (family A) exhibited disappearance of the maternal PGK RFLP and persistence of the paternal PGK RFLP, which is consistent with nonrandom inactivation of the paternal $\mathrm{X}$ chromosome. For HPRT, however, the opposite result was obtained: the maternal RFLP persisted and the paternal RFLP disappeared. A previous study used a replication banding technique to show that the paternal $\mathrm{X}$ chromosome was nonrandomly inactivated in this girl (1).

For the two patients with hemophilia A (families B and C), the results were identical to each other, but the converse of the hemophilia B patient (family A): the paternal PGK and maternal HPRT RFLPs disappeared and the maternal PGK and paternal HPRT RFLPs persisted. In the case of the fathers of the affected females, Hpa II digestion resulted in the disappearance of the PGK RFLP (Fig. 1), but Hha I (and Hpa II, not shown) digestion diminished the HPRT RFLP signal by $\sim 25 \%$ (Fig. 2), suggesting that in males the PGK sequence is unmethylated, whereas the HPRT sequence is relatively hypermethylated.
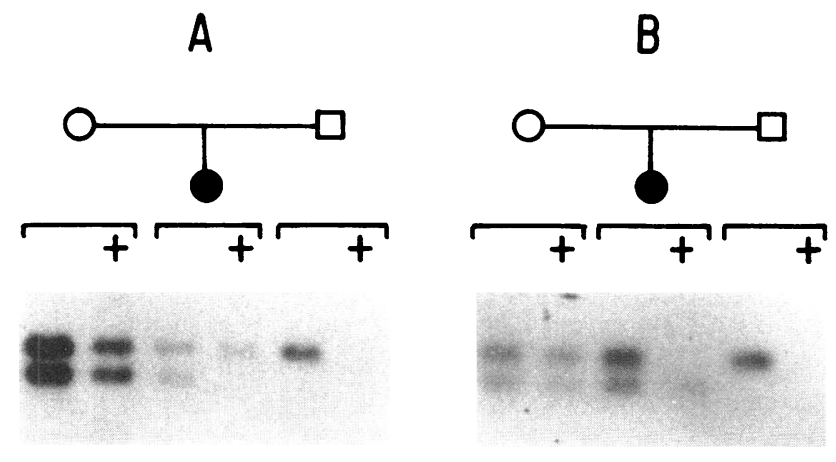

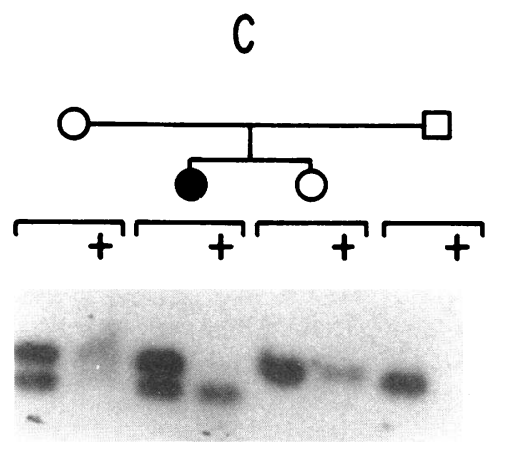

Figure 1. X chromosome methylation patterns using a PGK probe. $10 \mu \mathrm{g}$ of chromosomal DNA was digested to completion with Bst XI and Pst I restriction endonucleases. The sample was then split into two 5- $\mu \mathrm{g}$ aliquots, one of which was further digested to completion with Hpa II (+). The DNA samples were subjected to RFLP-Southern blot analysis as described in Methods. The pedigrees of the three families are indicated. 

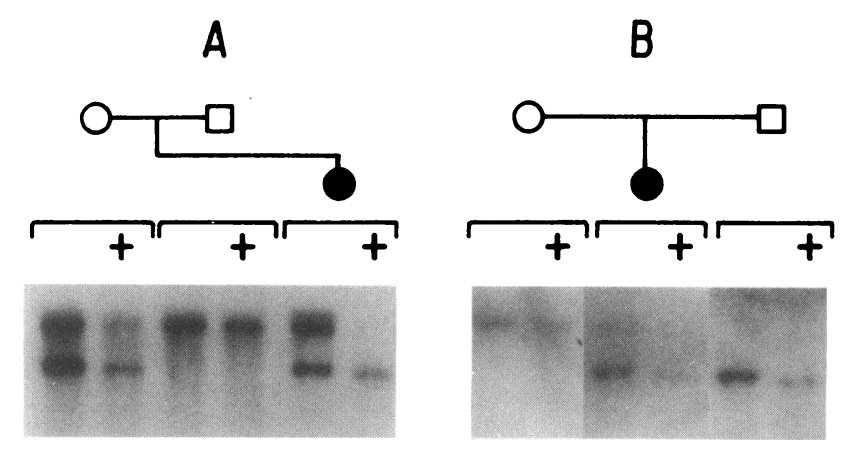

\section{Discussion}

DNA methylation has been implicated in a number of fundamental processes including cell differentiation (13), parental imprinting (14), and the control of gene expression (15). Variation in methylation of allelic sites on homologous autosomal chromosomes is tissue specific and reproducible after transmission through the germ line (16). Allele-specific methylation of the human c-Ha-ras-1 gene has also been described (17).

In $\mathrm{X}$ chromosome inactivation, regional intragenic patterns of methylation rather than ubiquitous differences may be important $(18,19)$. The $5^{\prime}$ region of the mouse HPRT gene is hypomethylated on the active $\mathrm{X}$ relative to the inactive $\mathrm{X}$, whereas a sequence in the middle of the gene is consistently hypomethylated in the inactive $X(20)$. Other regional differences in the methylation pattern of HPRT have also been described (21).

It is not clear whether DNA methylation is a primary event in the control of gene expression and $\mathrm{X}$ inactivation, or is a secondary phenomenon that maintains the pattern(s) of inactivation established by other mechanisms $(22,23)$. The recent observation that differential methylation of the HPRT gene occurs after $\mathrm{X}$ chromosome inactivation suggests that methylation does not play a role in the primary events of $X$ inactivation, but may function as part of a secondary, tissue-specific mechanism for maintaining the inactive state (24).

There is clearly a nonrandom pattern of $\mathrm{X}$ chromosome methylation in the PBL of our female patients with severe hemophilia. Since $\mathrm{X}$ chromosome inactivation and DNA methylation occur early in embryogenesis (24), it is reasonable to presume that lymphocytes and hepatocytes (which synthesize Factors VIII and IX) exhibit identical X chromosome methylation patterns. Therefore, these data strongly suggest that nonrandom $\mathrm{X}$ chromosome inactivation (lyonization) is the basis for severe hemophilia in females.

\section{Acknowledgments}

We graciously thank Dr. M. Hilgartner, Dr. D. Praeger, and Ms. A. Wolfer for providing blood specimens from their patients and their families, Dr. G. G. Brownlee for the Factor IX probes, Dr. Richard Lawn for the Factor VIII probes, Dr. J. Mandel for the St 14 probe, and Dr. K. Davies for the Dx 13 probe.

Dr. Nisen was supported by a Basil O'Connor Starter Scholar Research Award from the March of Dimes and a grant from the Elsa U. Pardee Foundation.

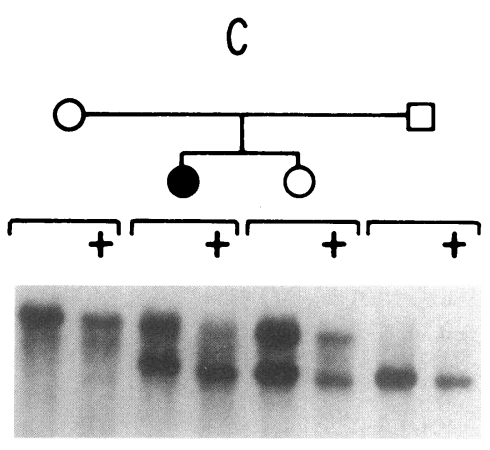

Figure 2. X chromosome methylation patterns using an HPRT probe. The same procedure as described in Fig. 1 was used. However, in this case the DNA was digested with Bam $\mathrm{H} 1$ restriction endonuclease followed by Hha I, and an HPRT probe was used.

\section{References}

1. Nisen, P., J. Stamberg, R. Ehrenpreis, S. Velasco, A. Shende, J. Engelberg, G. Karayalcin, and L. Waber. 1986. The molecular basis of severe hemophilia B in a girl. N. Engl. J. Med. 315:1139-1142.

2. Vogelstein, B., E. Fearon, S. Hamilton, and A. Feinberg. 1985. Use of restriction fragment length polymorphisms to determine the clonal origin of human tumors. Science (Wash. DC). 277:621-625.

3. Fearon, E., J. A. Winkelstein, C. I. Civin, D. M. Pardoll, and B. Vogelstein. 1985. Carrier detection in X-linked agammaglobulinemia by analysis of X-chromosome inactivation. $N$. Engl. J. Med. 316:427431.

4. Kohn, D., E. R. Fearon, J. A. Winkelstein, B. Vogelstein, and R. M. Blaese. 1987. Wiskott-Aldrich syndrome (WAS) carrier detection by X-chromosome inactivation analysis. Pediatr. Res. 21:313A. (Abstr.)

5. Conley, M., A. Lavoie, C. Briggs, P. Brown, C. Guerra, and J. M. Puck. 1988. Non-random X-chromosome inactivation in B cells from carriers of X-chromosome linked severed combined immunodeficiency. Proc. Natl. Acad. Sci. USA. 85:3090-3094.

6. Anson, D. S., K. H. Choo, D. J. Rees, F. Giannelli, J. A. Huddleston, and G. G. Brownlee. 1984. The gene structure of human anti-haemophilic factor IX. EMBO (Eur. Mol. Biol. Organ.) J. 3:1053-1060.

7. Gitschier, J., D. Drayna, E. G. Tuddenham, R. L. White, and R. M. Lawn. 1985. Genetic mapping and diagnosis of hemophilia A achieved through a Bcl I polymorphism in the factor VIII gene. Nature (Lond.). 314:738-740.

8. Oberle, I., G. Camerino, G. Heilig, L. Grunebaum, J. P. Cazenave, C. Crapanzano, P. M. Manucci, and J. L. Mandel. 1985. Genetic screening for hemophilia A with a polymorphic DNA probe. $N$. Engl. J. Med. 312:682-686.

9. Harper, K., R. M. Winter, M. E. Pembrey, D. Hartley, K. E. Davies, and E. G. Tuddenham. 1984. A clinically useful DNA probe closely linked to hemophilia A. Lancet. ii:6-8.

10. Singer-Sam, J., D. H. Keith, K. Tani, R. L. Simmer, L. Shively, S. Lindsay, A. Yoshida, and A. D. Riggs. 1984. Sequence of the promoter region of the gene for human X-linked 3-phosphoglycerate kinase. Gene (Amst.). 32:409-417.

11. Jolly, D., A. C. Esty, H. U. Bernard, and T. Friedmann. 1982. Isolation of a genomic clone partially encoding human hypoxanthine phosphoribosyl transferase. Proc. Natl. Acad. Sci. USA. 79:5038-5041.

12. Nisen, P., K. Zimmerman, S. Cotter, F. Gilbert, and F. Alt. 1986. Enhanced expression of the N-myc gene in Wilms' tumors. Cancer Res. 46:6217-6222.

13. Razin, A., C. Webb, M. Szyf, J. Yisraeli, A. Rosenthal, T. Naveh-Many, N. Sciaky-Gallili, and H. Cedar. 1984. Variations in DNA methylation during mouse cell differentiation in vivo and in vitro. Proc. Natl. Acad. Sci. USA. 81:2275-2279. 
14. Reik, W., A. Collick, M. L. Norris, S. C. Barton, and M. A. Surani. 1987. Genomic imprinting determines methylation of parental alleles in transgenic mice. Nature (Lond.). 328:248-251.

15. Cedar, H. 1988. DNA methylation and gene activity. Cell. 53:3-4.

16. Silva, A., and R. White. 1988. Inheritance of allelic blueprints for methylation patterns. Cell. 54:145-152.

17. Chandler, L., H. Ghazi, P. A. Jones, P. Boukamp, and N. E. Fusenig. 1987. Allele-specific methylation of the human c-Ha-ras-1 gene. Cell. 50:711-717.

18. Lyon, M. 1988. X-chromosome inactivation and the location and expression of X-linked genes. Am. J. Hum. Genet. 42:8-16.

19. Wolf, S., and B. Migeon. 1982. Studies of X-chromosome DNA methylation in normal human cells. Nature (Lond.). 295:667-671.
20. Yen, P., P. Patel, A. C. Chinault, T. Mohandas, and L. J. Shapiro. 1984. Differential methylation of HPRT genes on active and inactive X-chromosomes. Proc. Natl. Acad. Sci. USA. 81:1759-1763.

21. Wolf, S. F., D. J. Jolly, K. D. Lunnen, I. Friedmann, and B. R. Migeon. Methylation of the HPRT locus on the X chromosome: implications for X-chromosome inactivation. Proc. Natl. Acad. Sci. USA. 81:2806-2810.

22. Razin, A., and A. Riggs. 1980. DNA methylation and gene function. Science (Wash. DC). 210:604-610.

23. Bird, A. 1986. CpG rich islands and the function of DNA methylation. Nature (Lond.). 321:209-213.

24. Lock, L., N. Takagaki, and G. Martin. 1987. Methylation of the HPRT gene on the inactive $\mathrm{X}$ occurs after chromosome inactivation. Cell. 48:39-46. 